\title{
Commercial Vehicles as a Driving Force
}

\section{Dear Reader,}

The IAA Commercial Vehicles in Hanover receives little attention from the general public, but is of huge significance for its target audience. This issue of ATZ takes this into consideration in its cover feature with a report on the EU Ecochamps project. The project involves the development of five hybrid vehicles with the aim of using standardised drive and energy management systems across different vehicle segments, from cars and vans through to articulated trucks. MTZ 10/2016 also investigates the Ecochamps project from the perspective of the components of the drive system. Equally interesting features in this issue are the article on the flywheel energy storage system for buses and the interview with Dr. Carsten Intra from MAN, which covers the results of the gigaliner tests and the future of car-to-x communication in commercial vehicles.

The new generation of assistance systems, which lays the foundations for highly and fully autonomous driving, is pushing existing electric and electronic architectures to their limits. Systems for mediumsized and heavy-duty trucks, such as traffic jam assist and lane departure warning or pairing and platooning, require redundant communication concepts and system functions, but also increase the demands on the power supply in the vehicle. Future-proof electric and electronic architectures must be able to support innovative functions, including autonomous and electric driving, cyber security and predictive energy management.

There are currently clear indications that the commercial vehicle sector could act as the enabler for autonomous driving, because in addition to road safety, the total cost of ownership is a key driver of the rapid development and introduction of this technology. The behaviour of long-haul trucks is also more predictable than that of cars, which makes connectivity between vehicles easier to achieve. The first legislative changes are already underway and it will soon be possible to replace conventional rear view mirrors with camera systems. These systems offer huge potential for commercial vehicles, in particular in terms of safety, because they will undoubtedly help drivers to evaluate what is happening on the road more effectively. The display of a camera and monitor system can give drivers the information they need about the traffic situation at precisely the right moment.

It will be interesting to see which innovations in this area are presented at this year's IAA Commercial Vehicles.

Best wishes,

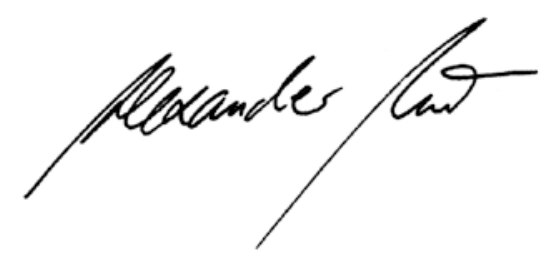

Dr. Alexander Heintzel

Editor in Chief

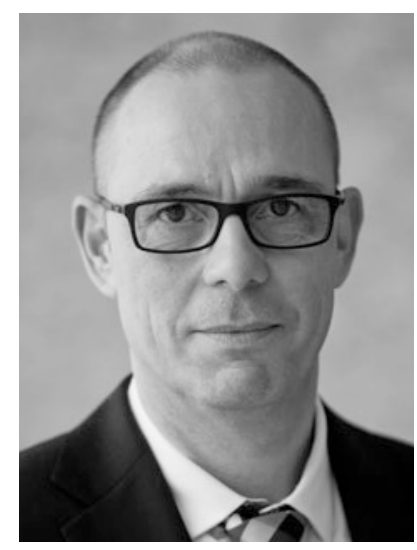

